\title{
Experimental and Theoretical Studies of Self-Organization of Complex Protein Structures
}

\author{
A. I. Evseev, O. V. Batishchev, and A. V. Indenbom \\ Frumkin Institute of Physical Chemistry and Electrochemistry of the Russian Academy of Sciences, Moscow; \\ e-mail: daspublishing@list.ru
}

DOI: $10.1134 / \mathrm{S} 1990747809030192$

Virus infection is a multistage process that starts with endocytosis followed by an exit of the viral genetic material into the cell cytoplasm. A lot of proteins participate in the process of endocytosis. At the last stages of endocytosis the membrane neck is represented by a membrane nanotube (NT) with luminal radius of a few nanometers. A set of GTPase dynamines is responsible for the NT fission. After the NT fission, virus-containing vesicle fuses with endosome, which moves to a cell nucleus along microtubules. Subsequent decrease of $\mathrm{pH}$ in endosome results in an activation of haemagglutinines (HA). Their conformational rearrangement is followed by self-organization of the protein complex resulting in a formation of fusion pore. The virus RNA exit into the cytoplasm occurs after destruction of M1 protein carcass surrounding nucleoproteins. Apparently, M1 proteins interact with each other as well as with ribonucleoproteins and lipid bilayer in a $\mathrm{pH}-$ dependent way. Self-organization of proteins in com- plex structures is mediated by the lipid component of the membranes. In particular, it is shown that proteins in the membrane are collected in extensive aggregates, so-called "islands", and the part of the membrane in these aggregates is in a liquid-ordered (raft) state. As we suppose, the appearance of rafts can be stipulated by the process of self-organization of protein-lipid systems and involve the mechanism of protein wetting by lipids. A loss of the membrane integrity can be produced by various factors. It always starts with formation of lipid or lipid-protein pores and leads to the cell death. Progressing of the pores can be promoted by an electric field, osmotic stress, or chemical modification of the membrane by the change of lipid composition or formation of lipid-protein structures. Usage of modern methods and approaches has allowed a considerable progress in understanding the virus infection and associated processes of self-organization of complex protein and lipid-protein structures. 